PROCEEDINGS OF THE

AMERICAN MATHEMATICAL SOCIETY

Volume 126, Number 11, November 1998, Pages 3371-3376

S 0002-9939(98)04305-6

\title{
HOMOGENEOUS IDEALS IN WICK $*$-ALGEBRAS
}

\author{
DANIIL PROSKURIN \\ (Communicated by Palle E. T. Jorgensen)
}

\begin{abstract}
The necessary and sufficient condition for the family of homogeneous elements to determine a Wick ideal is presented. The structure of homogeneous Wick ideals with degree higher than 2 is discussed. For the braided operator $T$ a formula to calculate the largest cubic ideal when the quadratic one is known is obtained. Irreducible $*$-representations of the $\mu$-CAR algebra are classified.
\end{abstract}

\section{INTRODUCTION}

In the study of *-representations of Wick algebras, it is useful to know the structure of Wick ideals, and especially, as examples show [1], of homogeneous Wick ideals. For example, in any bounded representation of $\mu$-CCR or $q_{i j}$-CCR, $\left|q_{i j}\right|=1$ as $i \neq j$, any quadratic ideal vanishes.

In the paper [1], the main attention was concentrated on quadratic ideals, and there was presented a necessary and sufficient condition imposed on the system of elements to generate a quadratic ideal.

In this note this criterion is extended to the general case (Sec. 2). In the case when the operator $T$ (see Sec. 1) satisfies the braid relation, and $-1 \leq T \leq 1$, we investigate the structure of homogeneous Wick ideals of higher degrees. In particular, we improve the theorem on strict positivity of the Fock representation, and derive a formula, which allows one to calculate a cubic ideal providing that a quadratic one is known (Sec. 3). In Sec. 4 these results are illustrated in the example of $\mu$-CAR algebra.

\section{Preliminaries}

Let $I=\{1, \ldots, d\}$, and $T_{i j}^{k l} \in \mathbb{C}, i, j, k, l \in I$, be such that $T_{i j}^{k l}=\bar{T}_{j i}^{l k}$. The Wick algebra with coefficients $\left\{T_{i j}^{k l}\right\}$ (see [1]) is a *-algebra generated by the elements $a_{i}$, $a_{i}^{*}$ and the defining relations

$$
a_{i}^{*} a_{j}=\delta_{i j} 1+\sum_{k, l=1}^{d} T_{i j}^{k l} a_{l} a_{k}^{*} .
$$

Denote by $\mathcal{H}=\left\langle e_{1}, \ldots, e_{d}\right\rangle$ the finite-dimensional space over $\mathbb{C}$, and by $\mathcal{H}^{*}$ its formal dual. $\mathcal{T}\left(\mathcal{H}, \mathcal{H}^{*}\right)$ will denote the tensor algebra over $\mathcal{H}, \mathcal{H}^{*}$. Then $\mathcal{W}$ can be

Received by the editors November 21, 1996 and, in revised form, December 10, 1996.

1991 Mathematics Subject Classification. Primary 81R50, 47A62, 46L05.

This work was partially supported by the CRDF, grant no. 292.

(C)1998 American Mathematical Society 
canonically realized as

$$
\mathcal{T}\left(\mathcal{H}, \mathcal{H}^{*}\right) /\left\langle e_{i}^{*} \otimes e_{j}-\delta_{i j} 1-\sum T_{i j}^{k l} e_{l} \otimes e_{k}^{*}\right\rangle .
$$

In this realization, the subalgebra, generated by $\left\{a_{i}\right\}$ is identified with $\mathcal{T}(\mathcal{H})$.

It is obvious that any element of $\mathcal{W}$ can be uniquely represented as a polynomial in the noncommuting variables $a_{i}, a_{i}^{*}$, where in each monomial, variables $a_{i}$ are placed to the left from $a_{j}^{*}$. Such monomials are called Wick ordered ones, and they form a basis in $\mathcal{W}$.

When studying properties of $\mathcal{W}$, one can find useful the following operators (see [1]):

$$
\begin{aligned}
& T: \mathcal{H} \otimes \mathcal{H} \mapsto \mathcal{H} \otimes \mathcal{H}, \quad T e_{k} \otimes e_{l}=\sum_{i, j} T_{i k}^{l j} e_{i} \otimes e_{j}, \\
& T_{i}: \mathcal{H}^{\otimes n} \mapsto \mathcal{H}^{\otimes n}, \quad T_{i}=\underbrace{1 \otimes \cdots \otimes 1}_{i-1} \otimes T \otimes \underbrace{1 \otimes \cdots \otimes 1}_{n-i-1}, \\
& R_{n}: \mathcal{H}^{\otimes n} \mapsto \mathcal{H}^{\otimes n}, \quad R_{n}=1+T_{1}+T_{1} T_{2}+\cdots+T_{1} T_{2} \cdots T_{n-1}, \\
& P_{n}: \mathcal{H}^{\otimes n} \mapsto \mathcal{H}^{\otimes n}, \quad P_{2}=R_{2}, P_{n+1}=\left(1 \otimes P_{n}\right) R_{n+1} .
\end{aligned}
$$

In what follows, we will use the commutation rule in $\mathcal{W}$ between $e_{i}^{*}$ and $X$, where $X \in \mathcal{H}^{\otimes n}$.

Proposition 1. Let $X \in \mathcal{H}^{\otimes n}$; then

$$
e_{i}^{*} \otimes X=\mu\left(e_{i}^{*}\right) R_{n} X+\mu\left(e_{i}^{*}\right) \sum_{k=1}^{d} T_{1} T_{2} \cdots T_{n}\left(X \otimes e_{k}\right) e_{k}^{*},
$$

where $\mu\left(e_{i}^{*}\right): \mathcal{T}(\mathcal{H}) \mapsto \mathcal{T}(\mathcal{H})$ is defined as follows:

$$
\mu\left(e_{i}^{*}\right) 1=0, \quad \mu\left(e_{i}^{*}\right) e_{i_{1}} \otimes \cdots \otimes e_{i_{n}}=\delta_{i i_{1}} e_{i_{2}} \otimes \cdots \otimes e_{i_{n}} .
$$

Proof. It follows from the defining relations that $e_{i}^{*} \otimes X \in \mathcal{H}^{\otimes n-1} \oplus \mathcal{H}^{\otimes n} \otimes \mathcal{H}^{*}$. The fact that the term $e_{i}^{*} \otimes X$ from $\mathcal{H}^{\otimes n-1}$ is equal to the first term in the formula (1) is essentially contained in [1], Lemma 2.1.1.

Now we prove that the term belonging to $\mathcal{H}^{\otimes n} \otimes \mathcal{H}^{*}$ is equal to the second term. It is obvious that one can assume $X=e_{i_{1}} \otimes e_{i_{2}} \otimes \cdots \otimes e_{i_{n}}$. Denote by $F_{i}$ the component of $e_{i}^{*} \otimes X$ which belongs to $\mathcal{H}^{\otimes n} \otimes \mathcal{H}^{*}$. Consider "deriving function" $F=\sum_{i=1}^{d} e_{i} \otimes F_{i}$. It is evident that $F_{i}=\mu\left(e_{i}^{*}\right) F$. The relations imply

$$
F=\sum_{i} \sum_{k_{1}, l_{1}} \cdots \sum_{k_{n}, l_{n}} T_{i i_{1}}^{k_{1} l_{1}} T_{k_{1} i_{2}}^{k_{2} l_{2}} \cdots T_{k_{n-1} i_{n}}^{k_{n} l_{n}} e_{i} \otimes e_{l_{1}} \otimes \cdots \otimes e_{l_{n}} \otimes e_{k_{n}}^{*} .
$$

Taking into account the definition of $T_{i}$ and changing summation order, we get

$$
F=\sum_{k_{n}} T_{1} \cdots T_{n}\left(e_{i_{1}} \otimes \cdots \otimes e_{i_{n}} \otimes e_{k_{n}}\right) \otimes e_{k_{n}}^{*},
$$

which completes the proof.

\section{Homogeneous ideals. General Case}

A Wick ideal (see [1]) is a two-sided ideal $I \subset \mathcal{T}(\mathcal{H})$, such that $\mathcal{T}\left(\mathcal{H}^{*}\right) \subset I \mathcal{T}\left(\mathcal{H}^{*}\right)$. If $I$ is generated by a set $I_{0} \subset \mathcal{H}^{\otimes n}$, then $I$ is called a homogeneous Wick ideal of degree $n$.

The following statement is a generalization of the fact established in [1] for $n=2$. 
Proposition 2. Let $P: \mathcal{H}^{\otimes n} \mapsto \mathcal{H}^{\otimes n}$ be a projection. Then $I_{n}=\left\langle P \mathcal{H}^{\otimes n}\right\rangle$ is a Wick ideal if and only if

1. $R_{n} P=0$,

2. $[1 \otimes(1-P)] T_{1} T_{2} \cdots T_{n}[P \otimes 1]=0$.

Moreover, if $T$ satisfies the braid condition $T_{1} T_{2} T_{1}=T_{2} T_{1} T_{2}$ and $P$ is a projection on $\operatorname{ker} R_{n}$, then the condition 2 holds automatically.

Proof. It follows from [1], Lemma 3.1.1 that $P$ generates a homogeneous ideal if and only if $\forall i=1, \ldots, d, \forall X \in \mathcal{H}^{\otimes n}$, the relation

$$
e_{i}^{*} \otimes P X \in P\left(\mathcal{H}^{\otimes n}\right) \otimes \mathcal{H}^{*}
$$

holds.

Then we have

$$
e_{i}^{*} \otimes P X=\mu\left(e_{i}^{*}\right)\left(R_{n} P X+\sum_{k=1}^{d} T_{1} \cdots T_{n}\left(P X \otimes e_{k}\right) \otimes e_{k}^{*}\right) .
$$

Since $\mu\left(e_{i}^{*}\right) R_{n} P X \in \mathcal{H}^{\otimes(n-1)}$, we have $\mu\left(e_{i}^{*}\right) R_{n} P X=0 \forall i, X$, which implies $R_{n} P=0$. Further, $\mu\left(e_{i}^{*}\right) \sum T_{1} \cdots T_{n}\left(P X \otimes e_{k}\right) \otimes e_{k}^{*} \in P\left(\mathcal{H}^{\otimes n}\right) \otimes \mathcal{H}^{*}$ if and only if $\forall k \mu\left(e_{i}^{*}\right) T_{1} \cdots T_{n}\left(P X \otimes e_{k}\right) \in P\left(\mathcal{H}^{\otimes n}\right)$, i.e., if

$$
\begin{gathered}
(1-P) \mu\left(e_{i}^{*}\right) T_{1} \cdots T_{n}(P \otimes 1)\left(X \otimes e_{k}\right)=0, \\
\mu\left(e_{i}^{*}\right)(1 \otimes(1-P)) T_{1} \cdots T_{n}(P \otimes 1)\left(X \otimes e_{k}\right)=0 .
\end{gathered}
$$

Since the equality holds $\forall i, k, X$, we have

$$
[1 \otimes(1-P)] T_{1} \cdots T_{n}[P \otimes 1]=0 .
$$

Now, let $T$ satisfy the braid condition. Then it is easy to check that $\forall k=1$, $\ldots, n-1$ we have $T_{1} T_{2} \cdots T_{n} T_{k}=T_{k+1} T_{1} T_{2} \cdots T_{n}$, which implies

$$
T_{1} T_{2} \cdots T_{n}\left(R_{n} \otimes 1\right)=\left(1 \otimes R_{n}\right) T_{1} T_{2} \cdots T_{n} .
$$

Therefore, if $X \in \operatorname{ker} R_{n}$, then $\forall k$ we have

$$
\left(1 \otimes R_{n}\right) T_{1} \cdots T_{n}\left(X \otimes e_{k}\right)=T_{1} \cdots T_{n}\left(R_{n} \otimes 1\right)\left(X \otimes e_{k}\right)=0 .
$$

The proof is done.

\section{Homogeneous ideals. Braided CASE}

In this section, we assume that $T$ satisfies the braid relation, and $-1 \leq T \leq 1$. Under these conditions, the maximal quadratic ideal $I_{2}$ is generated by $\operatorname{ker}(1+T)$. It is obvious that if $-1<T \leq 1$, then $I_{2}=\{0\}$. It appears that in this case $\forall n \geq 2$, $I_{n}=\{0\}$.

Proposition 3. If $-1<T \leq 1$, and $T_{1} T_{2} T_{1}=T_{2} T_{1} T_{2}$, then $\operatorname{ker} R_{n}=\{0\}$ for all $n \geq 2$.

Remark 1. This statement is an elaboration of Theorem 2.3 in [2] for the case $S_{n}$.

If $-1 \leq T<1$, then one can prove that $I_{n} \subset I_{2}$. For $n=3$, a more precise statement holds.

Theorem 1. If $T_{1} T_{2} T_{1}=T_{2} T_{1} T_{2}$ and $-1 \leq T \leq 1$, then

$$
\text { ker } R_{3}=\left(1-T_{1} T_{2}\right)\left(\operatorname{ker} R_{2} \otimes \mathcal{H}\right) \text {; }
$$

in particular, $I_{3} \subset I_{2}$. 
Proof. We recall that $R_{2}=1+T$; then $\operatorname{ker} R_{2} \otimes \mathcal{H}=\operatorname{ker}\left(1+T_{1}\right), \mathcal{H} \otimes \operatorname{ker} R_{2}=$ $\operatorname{ker}\left(1+T_{2}\right)$. Consider operator $P_{3}=\left(1 \otimes R_{2}\right) R_{3}$; then it is obvious that ker $R_{3} \subset$ $\operatorname{ker} P_{3}$. We prove that $\operatorname{ker} P_{3}=\operatorname{ker}\left(1+T_{1}\right)+\operatorname{ker}\left(1+T_{2}\right)$. To do it, we need the following formulas:

$$
\begin{gathered}
P_{3}=\left(1+T_{2}\right)\left(1+T_{1}+T_{1} T_{2}\right), \\
P_{3}=\left(1+T_{1}\right)\left(1+T_{2}+T_{2} T_{1}\right), \\
R_{3}=1+T_{1}+T_{1} T_{2}, \hat{R}_{3}=1+T_{2}+T_{2} T_{1} .
\end{gathered}
$$

These formulas demonstrate that $R_{3}, \hat{R}_{3}$ : $\operatorname{ker} P_{3} \mapsto \operatorname{ker} P_{3}$, and $R_{3}$ maps ker $P_{3}$ on $\operatorname{ker}\left(1+T_{2}\right), \hat{R}_{3}$ projects on $\operatorname{ker}\left(1+T_{1}\right)$. We denote by $R$ the restriction of the operator $R_{3}+\hat{R}_{3}$ to $\operatorname{ker} P_{3}$. Since $R=R^{*}$, we have $\operatorname{ker} P_{3}=\operatorname{im} R \oplus \operatorname{ker} R$, and moreover $\operatorname{im} R \subset \operatorname{ker}\left(1+T_{1}\right)+\operatorname{ker}\left(1+T_{2}\right)$. Let $Y \in \operatorname{ker} R$. Since $R_{3}+\hat{R}_{3}=$ $P_{3}+1-T_{1} T_{2} T_{1}$ and $Y \in \operatorname{ker} P_{3}$, we have $\left(1-T_{1} T_{2} T_{1}\right) Y=0$. The latter equality holds if and only if the following equalities hold:

$$
\begin{aligned}
& \left(1-T_{1}^{2}\right) Y+T_{1}\left(1-T_{2}\right) T_{1} Y=0 \\
& \left(1-T_{2}^{2}\right) Y+T_{2}\left(1-T_{1}\right) T_{2} Y=0
\end{aligned}
$$

Taking into account the condition $-1 \leq T \leq 1$, we get

$$
\left(1-T_{1}^{2}\right) Y=0, \quad\left(1-T_{2}^{2}\right) Y=0, \quad T_{2} T_{1} Y=T_{1} Y, \quad T_{1} T_{2} Y=T_{2} Y .
$$

The condition $P_{3} Y=0$ takes the form $\left(1+T_{1}+T_{2}\right) Y=0$. Now we can easily check that

$$
\left(1-T_{1}\right) Y \in \operatorname{ker}\left(1+T_{1}\right), \quad\left(2+T_{1} Y\right) \in \operatorname{ker}\left(1+T_{2}\right),
$$

which implies that $Y \in \operatorname{ker}\left(1+T_{1}\right)+\operatorname{ker}\left(1+T_{2}\right)$.

Therefore, ker $R \subset \operatorname{ker}\left(1+T_{1}\right)+\operatorname{ker}\left(1+T_{2}\right)$, and thus $\operatorname{ker} P_{3}=\operatorname{ker} R_{2} \otimes \mathcal{H}+$ $\mathcal{H} \otimes \operatorname{ker} R_{2}$.

Since the restriction of $R_{3}$ to $\operatorname{ker} P_{3}$ is a projection, and $\operatorname{ker} R_{3} \subset \operatorname{ker} P_{3}$, we have $\operatorname{ker} R_{3}=\left(1-R_{3}\right)\left(\operatorname{ker} P_{3}\right)$. It is easy to check that $\left(1-R_{3}\right)\left(\operatorname{ker} P_{3}\right)=$ $\left(1-T_{1} T_{2}\right)\left(\operatorname{ker} R_{2} \otimes \mathcal{H}\right)$.

\section{4. *-REPRESEntations of $\mu$-CAR ALGEBRA}

Here we consider $*$-representations of the $\mu$-CAR algebra by the Hilbert space operators. This algebra was introduced in [1] as the algebra generated by the elements $a_{i}, a_{i}^{*}, i=1, \ldots, d$, which satisfy the following relations:

$$
\begin{aligned}
& a_{i}^{*} a_{i}=1-a_{i} a_{i}^{*}-\left(1-\mu^{2}\right) \sum_{k<i} a_{k} a_{k}^{*}, \\
& a_{i}^{*} a_{j}=-\mu a_{j} a_{i}^{*}, \quad 0<\mu<1 .
\end{aligned}
$$

It is easy to see that any $*$-representation of $\mu$-CAR is bounded.

To classify $*$-representations of any Wick algebra it is useful to investigate the Wick ideal structure of this algebra, and especially to describe quadratic Wick ideals (see [1]). For $\mu$-CAR the largest quadratic ideal

$$
I_{2}=\left\langle a_{i}^{2}, i=1, \ldots, d ; a_{j} a_{i}+\mu a_{i} a_{j}, i<j\right\rangle
$$

is very large; we consider a smaller quadratic ideal

$$
\hat{I}_{2}=\left\langle a_{i}^{2}, i=1, \ldots, d-1 ; a_{j} a_{i}+\mu a_{i} a_{j}, i<j\right\rangle .
$$

The main result of this note is the following theorem: 
Theorem 2. The ideal $\hat{I}_{2}$ vanishes in any representation of the $\mu$-CAR algebra.

Proof. Let us denote $A=a_{1}^{2}, B=a_{2} a_{1}+\mu a_{1} a_{2}$. It follows from the basic relations that

$$
\begin{aligned}
& A^{*} A=A A^{*}, \\
& A^{*} a_{k}=\mu^{2} a_{k} A^{*}, \quad k>1 .
\end{aligned}
$$

By the Fuglede-Phutnam theorem we have that $A a_{k}=\mu^{2} a_{k} A, k>1$. (It is obvious that $A a_{1}=a_{1} A$.) It is easy to see, that these relations imply that in any irreducible representation either $A=0$ or $\operatorname{ker} A=\{0\}$. Suppose now that $\operatorname{ker} A=\{0\}$. Then we have

$$
\begin{gathered}
B^{*} B=\mu^{2} B B^{*}+\left(1-\mu^{4}\right)\left(1+\mu^{2}\right) A A^{*}, \\
A^{*} B=\mu^{2} B A^{*}, \quad A B=\mu^{2} B A .
\end{gathered}
$$

Since $A A^{*}>0$, we have $B^{*} B>0$. Let $\pi$ be an irreducible representation of $\mu$-CAR. Consider a polar decomposition $\pi\left(b^{*}\right)=W T$; here $T \geq 0$ and $W$ is coisometry. Using the unitary reduction we can represent $\pi(A), T, W^{*}$ in the following form:

$$
\begin{aligned}
\pi(A) & =\operatorname{diag}\left(\lambda \mu^{2 n} x 1, n=0,1,2, \ldots\right), \\
T & =\operatorname{diag}\left(T_{n}, n=0,1,2, \ldots\right), \\
T_{0} & =0, \\
T_{n} & =x\left(1+\mu^{2}\right) \mu^{n-1}\left(1-\mu^{2 n}\right)^{\frac{1}{2}}, \quad n \geq 1,
\end{aligned}
$$

$W^{*}$ is multiple of the unilateral shift. Since $a_{1} A=A a_{1}, a_{1}^{*} A=A a_{1}^{*}$, we have

$$
a_{1}=\operatorname{diag}\left(b_{i}, i=1,2, \ldots\right) \text {, }
$$

$B=T W^{*}$, and in the matrix form:

$$
B=\left(\begin{array}{cccc}
0 & & & \\
T_{1} & 0 & & \\
& T_{2} & 0 & \\
& & \ddots & \ddots
\end{array}\right) .
$$

The relation $a_{1}^{*} B=\mu B a_{1}^{*}$ takes the form:

$$
b_{n+1}^{*} T_{n}=\mu T_{n} b_{n}^{*}
$$

If $x \neq 0$, then $b_{k}=\mu^{k-1} b_{1}$, and from $a_{1}^{*} a_{1}=1-a_{1} a_{1}^{*}$ we have:

$$
\begin{aligned}
b_{1}^{*} b_{1} & =1-b_{1} b_{1}^{*}, \\
\mu^{2} b_{1} b_{1}^{*} & =1-\mu^{2} b_{1} b_{1}^{*} .
\end{aligned}
$$

The latter equations are compatible only in the case $\mu^{2}=1$. This implies that $x=0, B=0, A=0$.

Let us denote $B_{k}=a_{k} a_{1}+\mu a_{1} a_{k}, k>2$; then from the basic relations we have:

$$
B_{k}^{*} B_{k}=\mu^{2} B_{k} B_{k}^{*}+\mu^{2}\left(1-\mu^{2}\right) \sum_{1<i<k} B_{i} B_{i}^{*}+\left(1+\mu^{2}\right)\left(1-\mu^{4}\right) A A^{*} .
$$


It follows from the boundedness of $B_{k}$ that applying induction we obtain $B_{k}=0$, $k=3, \ldots, d$. Hence we have $a_{k} a_{1}+\mu a_{1} a_{k}=0$, and in the irreducible representation

$$
\begin{aligned}
& a_{1}=\left(\begin{array}{ll}
0 & 0 \\
1 & 0
\end{array}\right) \otimes 1 \\
& a_{k}=\left(\begin{array}{cc}
1 & 0 \\
0 & -\mu
\end{array}\right) \otimes \hat{a}_{k}, \quad k>1 .
\end{aligned}
$$

where $\left\{\hat{a}_{k}, k>1\right\}$ satisfy $\mu$-CAR with $d-1$ generators.

It is obvious that $a_{j}^{2}=0$ if and only if $\hat{a}_{j}^{2}=0$ and $a_{j} a_{i}+\mu a_{i} a_{j}=0$ if and only if its relation holds for $\hat{a}_{j}, \hat{a}_{i}$. Then the induction on $d$ completes the proof.

The author expresses his gratitude to Prof. Yu. S. Samollenko and Prof. V. Ostrovskyı̆.

\section{REFERENCES}

1. P. E. T. Jørgensen, L. M. Schmitt and R. F. Werner. Positive representations of general commutation relations allowing Wick ordering. J. Funct. Anal. 134, no. 1 (1995), 33-99. MR 96h: 81033

2. M. Bożejko and R. Speicher. Completely positive maps on Coxeter groups, deformed commutation relations, and operator spaces. Mat. Ann. 300 (1994), 97-120. MR 95g:46105

Department of Mathematics, Kiev University, Kiev, Ukraine

E-mail address: prosk@imath.kiev.ua 